\title{
Response of Mitochondrial Traffic to Axon Determination and Differential Branch Growth
}

\author{
Gordon Ruthel and Peter J. Hollenbeck \\ Department of Biological Sciences, Purdue University, West Lafayette, Indiana 47907-2504
}

\begin{abstract}
Mitochondria are concentrated in regions of the neuron where the demand for mitochondrial function is high, such as nodes of Ranvier, synapses, and active growth cones. Does mitochondrial transport respond to changes in neuronal energy consumption and architecture, or does it precede and perhaps predict them? We have used axon determination, elongation, and alternating branch growth in hippocampal neurons to analyze the cellular cues that control mitochondrial traffic. During the stage 2-3 transition, when one minor process becomes the axon and accelerates its growth, mitochondria do not uniformly cluster at the base of the prospective axon. There is increased entry of mitochondria into the nascent axon, but this does not require accumulation near the axon. After axonal elongation is under way, the mitochondrial density of the minor processes decreases. Axonal towing experiments showed that elongation alone does not result in transport of mitochondria into the axon; thus, cytoplasmic flow cannot explain the entry of mitochondria into growing axons. Analysis of mitochondrial transport during alternating growth of axonal branches showed that mitochondrial traffic responds to changes in growth through regulation of entry into, but not exit from, branches. Branch-towing experiments showed that this response is not caused by axonal elongation alone, nor does it require an active growth cone. We propose that mitochondrial traffic in axons responds to changes in axonal outgrowth, and that the mechanism by which sorting at branch points occurs is different from the mechanism responsible for concentrating mitochondria at the growth cone.
\end{abstract}

Key words: mitochondria; axonal transport; axon outgrowth; growth cone; polarity; hippocampal neurons

\section{Introduction}

The growth, maintenance, and survival of neurons rely critically on mitochondria. These organelles not only perform aerobic ATP synthesis and buffer cytoplasmic free calcium but also generate the majority of the reactive oxygen species of the neuron and play a role in apoptotic and necrotic cell death. Thus, failures of mitochondrial function are thought to underlie excitotoxic injury and diverse degenerative diseases of the nervous system (Ankarkrona et al., 1995; Kosel et al., 1999; Reynolds, 1999; Nicholls and Budd, 2000; Raha and Robinson, 2000; Sawa, 2001; Swerdlow and Kish, 2002). Because of the extreme dimensions and asymmetry of neurons, their demands for mitochondrial functions and products are distributed very heterogeneously over long distances that preclude efficient delivery by diffusion. The transport and distribution of mitochondria must therefore respond to changing regional metabolic requirements. For example, nodes of Ranvier (Berthold et al., 1993; Waxman and Ritchie, 1993), myelination boundaries (Bristow et al., 2002), active synapses or tracts (Wong-Riley and Welt, 1980; Kageyama and Wong-Riley, 1982; Bindokas et al., 1998), and the region of active

\footnotetext{
Received June 11, 2003; revised July 30, 2003; accepted Aug. 1, 2003.

This work was supported by National Institutes of Health Grant NS27073. We are grateful to Phillip Lamoureux and Steven Heidemann for help and advice in towing axons.

Correspondence should be addressed to Peter J. Hollenbeck, Department of Biological Sciences, Purdue University, Lilly Hall, 915 West State Street, West Lafayette, IN 47907-2504. E-mail: phollenb@bilbo.bio.purdue.edu.

G. Ruthel's present address: Department of Cell Biology and Biochemistry, United States Army Medical Research Institute of Infectious Diseases, 1425 Porter Street, Frederick, MD 21702.

Copyright $\odot 2003$ Society for Neuroscience $\quad$ 0270-6474/03/238618-07\$15.00/0
}

growth cones (Morris and Hollenbeck, 1993) all contain high concentrations of mitochondria compared with surrounding regions of the axon.

We are particularly interested in the coordination of mitochondrial movement with axonal outgrowth. The ATP produced by mitochondria is required for many events of outgrowth, including growth cone motility, organelle transport, and cytoskeletal assembly (Bernstein and Bamburg, 2003). In cultured neurons, changes in growth cone activity lead to specific regulation of mitochondrial motility. Mitochondria accumulate in the region of an active growth cone and evacuate the region of an inactive one through a transition between stationary and motile states, along with upregulation and downregulation of anterograde but not retrograde motor activity (Morris and Hollenbeck, 1993; Hollenbeck, 1996). What are the cellular cues to which mitochondrial traffic responds? What is the relationship between axon growth and mitochondrial traffic?

To address these questions, we have studied mitochondrial transport and sorting in hippocampal neurons developing in culture. Although previous studies indicated that specific changes in mitochondrial motility occur in response to changes in axonal growth (Morris and Hollenbeck, 1993), it has been suggested that in hippocampal neurons, mitochondrial clustering at the base of one of the minor processes precedes, predicts, and perhaps even determines the decision of which process becomes the axon (Bradke and Dotti, 1997; Mattson and Partin, 1999). It has also been proposed that mitochondrial entry into a growing axon may occur by a general cytoplasmic flow in response to tensile forces at 


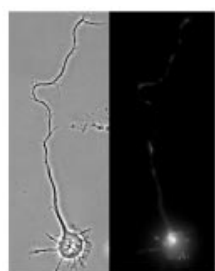

$A=0-45^{\circ}$

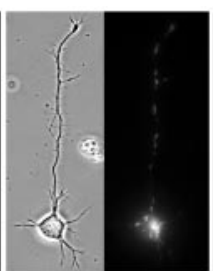

$B=46-90^{\circ}$

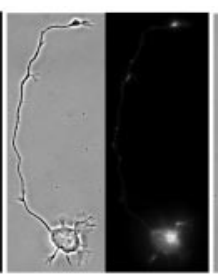

$C=91-135^{\circ}$

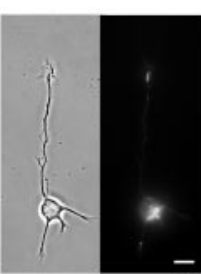

$D=136-180^{\circ}$

Figure 1. After the axon has been determined, mitochondria in the cell body show a modest tendency to cluster near its base. Paired phase contrast and R123 fluorescence images show examples of neurons with mitochondria clustered in the cell body in each of four radial bins measured relative to the position of the axon: $A, 0-45^{\circ}$ in either direction from the base of the axon; $B$, $46-90^{\circ} ; C, 91-135^{\circ}$; and $D, 136-180^{\circ}$. The accompanying histogram shows how frequently mitochondria in the cell body were seen to cluster within each radial bin ( $n=100$ neurons). The percentage of neurons without apparent mitochondrial clustering in their cell bodies is represented by the bar labeled None. Scale bar, $20 \mu \mathrm{m}$.

the growth cone (Bradke and Dotti, 1997). We have followed mitochondrial transport continuously in hippocampal neurons during axon determination and alternating growth of axon branches as they occur with a growth cone and also when the normal involvement of the growth cone is bypassed through experimentally applied tension. Our results are consistent with the view that mitochondrial motility responds to axon outgrowth rather than predicting it but suggest that control over mitochondrial movement in neurons occurs via a more specific mechanism than bulk cytoplasmic flow. We further demonstrate that different mechanisms serve to concentrate mitochondria in the axon tip versus sorting them at branch points.

\section{Materials and Methods}

Cell culture. Rat hippocampal neurons were cultured according to the protocol of Goslin and Banker (1991) with modifications that have been described previously (Ruthel and Hollenbeck, 2000). In short, hippocampi were dissected from embryonic day 18 rats and dissociated by treatment with $0.25 \%$ trypsin followed by trituration. Cells were plated onto poly-L-lysine-treated coverslips in MEM with $10 \%$ horse serum. After $\sim 4 \mathrm{hr}$, coverslips were inverted and transferred into culture dishes containing a glial monolayer and $\mathrm{CO}_{2}$-independent medium (Invitrogen, Grand Island, NY) with $2 \%$ glucose, $2 \mathrm{~mm}$ glutamine, $1 \mathrm{~mm}$ pyruvate, $0.1 \%$ ovalbumin, and the $\mathrm{N}_{2}$ supplements of Bottenstein and Sato (1979). The neurons on the coverslips were separated from the glial monolayer by wax dots. Aliquots of cells from each dissection were also cryogenically preserved at $-80^{\circ} \mathrm{C}$ in MEM with $10 \%$ horse serum and $8 \%$ dimethylsulfoxide before plating onto coverslips. When cells had reached the appropriate stage of development, coverslips were transferred into custom-made cell culture chambers. Chambers were then placed onto a microscope stage and heated to $36^{\circ} \mathrm{C}$ with an air curtain incubator (Nevtek, Burnsville, VA).

Image acquisition and analysis. Mitochondria were stained by treating cells with $1.0 \mu \mathrm{g} / \mathrm{ml}$ rhodamine 123 (R123) for 20-40 min. Images were acquired at intervals with either a Zeiss (Thornwood, NY) IM35 inverted microscope and a Hamamatsu (Hamamatsu City, Japan) enhanced CCD camera or a Nikon (Tokyo, Japan) TE300 inverted microscope and a Hamamatsu cooled CCD camera. In either case, image acquisition was controlled via Metamorph software (Universal Imaging, West Chester, PA). For experiments using the cooled CCD camera, phase contrast and fluorescence images were acquired in rapid succession with the aid of two Uniblitz shutters that allowed automated alternation between light sources.

Mitochondria entering or exiting branches were counted from video recordings. Lengths were measured using a calibrated measure function of the Metamorph software. Mitochondrial density was quantified by determining the projected area occupied by mitochondrial R123 fluorescence in specific regions of the axon using Metamorph software. Data from mitochondrial motility or density measurements were tested for normality and then compared using a paired Student's $t$ test.

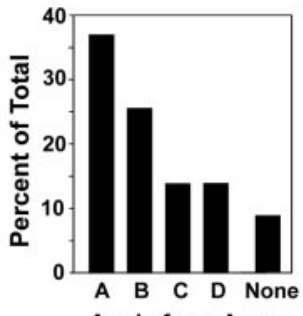

Angle from Axon
Modification of neurite outgrowth. The application of mechanical tension with a glass needle has been used to elicit the outgrowth of neurites from a variety of neurons (Bray, 1984; Lamoureux et al., 2002) To stimulate growth of a specific minor process on a stage 2 hippocampal neuron or of a nongrowing axon branch of a stage 3 neuron, we applied tension on the process using a glass needle in a manner similar to that of Lamoureux et al. (2002). Glass needles were coated with $0.1 \mathrm{mg} / \mathrm{ml}$ poly-L-lysine for 30-60 min then with $10 \mathrm{mg} / \mathrm{ml}$ concanavalin A (Sigma, St. Louis, MO) for $1 \mathrm{hr}$ or longer. Needles were placed in a holder on a micromanipulator attached to the microscope. After attachment of a neurite tip to the needle, tension was generated by moving the stage relative to the needle.

In experiments designed to separate process outgrowth from growth cone motility, we applied $0.2-0.4 \mu \mathrm{g} / \mathrm{ml} \mathrm{cy-}$ tochalasin E (Sigma) to the cells. Such treatment has previously been shown to disrupt growth cone motility without blocking outgrowth, axon determination, branch growth alternation (Ruthel and Hollenbeck, 2000), or mitochondrial motility (Morris and Hollenbeck, 1995).

\section{Results}

\section{Mitochondrial entry into nascent axons follows, rather than precedes, axonal outgrowth}

At stage 2 of their development in culture, hippocampal neurons have several equivalent undifferentiated minor processes that undergo unsynchronized cycles of extension and retraction. During the transition from stage 2 to stage 3 , this symmetry is disrupted when one process begins a rapid and sustained elongation to become the axon (Dotti et al., 1988; Ruthel and Hollenbeck, 2000). Although the specific signals and intracellular events that underlie the selection and development of one process into an axon are not completely clear, we expect sustained rapid growth to increase the energy demands of the nascent axon. Thus, it has been suggested that the concentration of somatic mitochondria at the base of one of the minor processes precedes and perhaps predicts its determination as the axon. To test this hypothesis, we used R123 staining to examine the position of mitochondria during and shortly after the stage $2-3$ transition.

The size of the nucleus constrains the distribution of mitochondria and other organelles (Dotti and Banker, 1991) toward the periphery of the soma, near the bases of the processes. We found that in $90 \%$ of stage 2-3 hippocampal neurons, most of the somatic mitochondria are located in a single cluster (Fig. 1). When we analyzed the distribution of mitochondria shortly after axon determination, we found that they do have a modest tendency to cluster in the region near the base of the axon. However, most $(64 \%)$ of the newly formed axons do not have a somatic mitochondrial cluster located within $\pm 45^{\circ}$ of their base. Given that the rapidly growing axon requires the delivery of mitochondria, how can this be the case? Continuous time-lapse observations of mitochondrial transport during the stage 2-3 transition demonstrated that mitochondria are efficiently delivered into the nascent axon regardless of where the somatic mitochondrial cluster is located, even when it is at the opposite end of the soma from the axon hillock (Fig. 2). Thus, early in its elongation, the nascent axon recruits mitochondria just like any other actively growing minor process (Fig. 2) or any elongating neurite (Morris and Hollenbeck, 1993).

It remained possible that a change in mitochondrial distribution does precede axon determination, such that a future axon 
receives a higher density of mitochondria than other processes even before it has committed to an axonal fate. To test this, we quantified the mitochondrial density of each neuronal process during the stage 2-3 transition. Three time points were selected on the basis of axon length: when the nascent axon was $(1)<5 \mu \mathrm{m}$ longer than the next longest process, a difference consistent with the nascent axon still being a minor process; (2) 15-20 $\mu \mathrm{m}$ longer than the next longest process, a difference indicating that a critical length may have been reached for axon determination; and (3) 35-60 $\mu \mathrm{m}$ longer, a difference that shows unambiguously that the process has been determined as an axon. We found that the mitochondrial density of the process that will become the axon does not exceed that of the other minor processes but, in fact, just the opposite; it has a lower mitochondrial density than the other processes (Fig. 3). Even at what is typically considered the critical length differential of axon formation, 15-20 $\mu \mathrm{m}$ longer than any other process, the nascent axon does not have a mitochondrial advantage over the other processes. This argues that mitochondria do not determine which process becomes the axon. Although sorting into the nascent axon must occur to maintain the mitochondrial density as length increases, this does not precede the onset of rapid growth. Not until after the axon has become $>35 \mu \mathrm{m}$ longer than the next longest process does the mitochondrial density become greater than that of the other processes (Fig. 3). Even then, the mitochondrial density of the axon exceeds that of the minor processes primarily because the latter diminishes by nearly $50 \%$, whereas the mitochondrial density of the axon shows only a modest increase (Fig. 3B). Together these results indicate that axonal determination and outgrowth are accompanied by changes in the transport and distribution of mitochondria, but these changes occur after, and probably in response to, axonal outgrowth.

\section{Sorting at axonal branch points delivers mitochondria to growing tips after growth status changes}

Another characteristic feature of the outgrowth of hippocampal axons is the alternation of periods of growth between sister branches of the same axon. The branches do not simultaneously elongate as they do in peripheral neurons (Lamoureux et al., 1998) but alternate periods of growth, such that one branch has an active growth cone and elongates, whereas the other remains quiescent; after a few hours, the growing branch ceases elongation, and the quiescent branch resumes growth (Ruthel and Banker, 1999; Ruthel and Hollenbeck, 2000).

Mitochondria move toward and accumulate in the distal region of actively growing unbranched peripheral axons (Morris and Hollenbeck, 1993). Unbranched hippocampal axons also show a distal accumulation of mitochondria during active growth (Figs. 1, 2), and we confirmed this quantitatively: the mitochondrial area in the distal-most $25 \mu \mathrm{m}\left[15.1 \pm 1.1(\mathrm{SEM}) \mu \mathrm{m}^{2}\right]$ of unbranched axons was significantly greater than in the adjacent $25 \mu \mathrm{m}\left(5.8 \pm 1.0 \mu \mathrm{m}^{2} ; n=26 ; p<0.001\right.$, paired $t$ test $)$. However, when an axon forms two branches, only one of which is growing, supplying mitochondria to the growing tip could involve not only regulation of the direction and duty cycle of movement but also specific sorting at the branch point. Studies in Aplysia neurons have shown that the volume of organelle traffic is greater in an axonal branch stimulated by a contact with a target cell than in

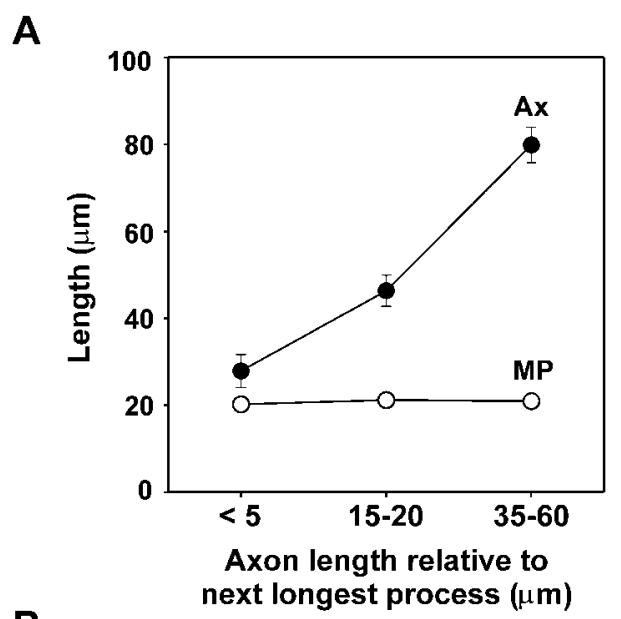

B

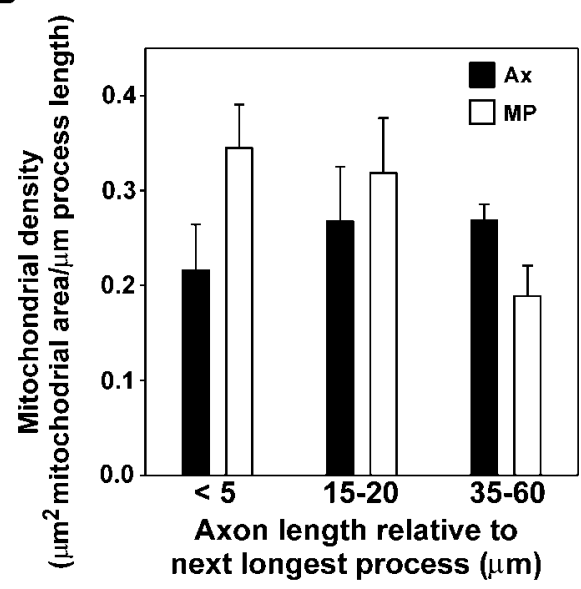

Figure 3. Mitochondrial transport into axons and minor processes is modulated after significant axonal outgrowth. $A$, Average lengths for the axon ( $A x$, filled circles) and minor processes (MP, open circles) are shown for three stages of axon formation analyzed: when the nascent axon is $<5 \mu \mathrm{m}$ longer than any other process, when it has reached a length $15-20 \mu \mathrm{m}$ greater than the next longest process, and when it has reached a length 35-60 $\mu \mathrm{m}$ greater in length than any of the remaining processes. Error bars indicate 1SEM; for minor process length, error bars do not extend beyond the circles. $B$, Separate measurements of the changes in mitochondrial density within the axon ( $A x$, filled bars) and those in minor processes (MP, open bars) during axon formation reveal that axonal mitochondrial density actually increases only slightly and not significantly ( $p=0.369$, paired $t$ test) as length increases. After the axon has been determined and has reached a length 35-60 $\mu \mathrm{m}$ greater than that of the next longest process, however, the minor processes show a significant drop in mitochondrial density ( $p=0.004$, paired $t$ test). Eight neurons were followed through the stage $2-3$ transition, and the mitochondrial density of every process was quantified for each. 

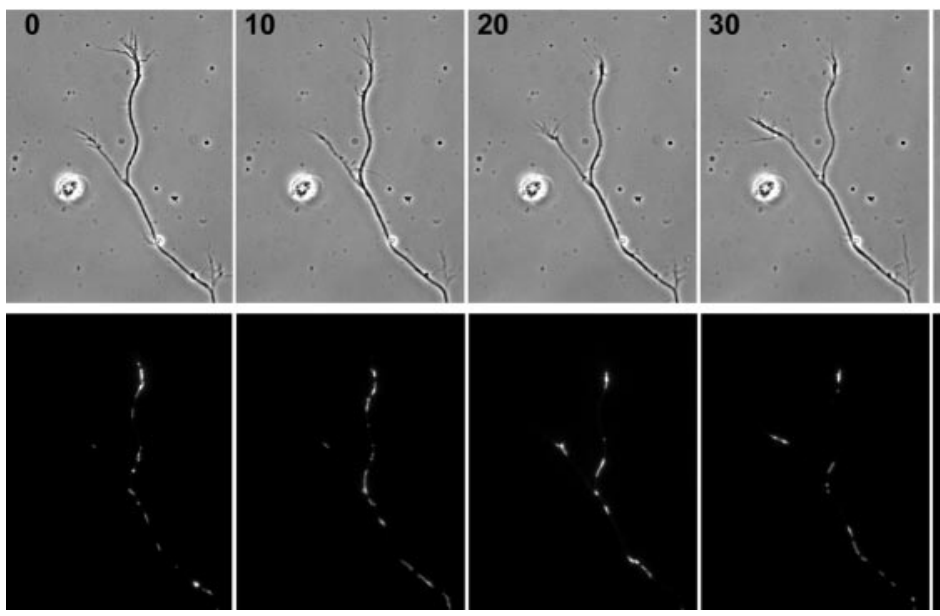

Figure 4. The density of mitochondria in axonal branches changes during alternation of branch growth. Phase contrast and corresponding R123 fluorescence images of a branched axon are shown at successive 10 min intervals. At the beginning of the series ( $0 \mathrm{~min})$, the right branch is growing and is filled with mitochondria, whereas the nongrowing left branch is relatively devoid of mitochondria. Between 10 and 20 min later, the right branch has stopped growing, and the left branch has begun to grow. Likewise, mitochondria have begun to vacate the right branch and have begun to enter the left branch. Sorting of mitochondria continues to be predominantly into the left branch as this branch continues to grow. Scale bar, $20 \mu \mathrm{m}$.

Table 1. Sorting of mitochondria in branched axons

\begin{tabular}{llll}
\hline & Growing & Nongrowing & Paired $t$ test \\
\hline Into branch & 7.15 & 3.23 & $p=0.024$ \\
Out of branch & 2.95 & 4.18 & $p=0.320$ \\
Paired $t$ test & $p=0.003$ & $p=0.414$ & $n=14$ cells \\
\hline
\end{tabular}

Mitochondria are selectively sorted into growing branches but do not selectively exit from nongrowing branches. The numbers of mitochondria moving through axonal branch points per hour were measured for the four possible classes of transport: mitochondria moving out of the main axon and into the growing branch, those moving from the main axon into the nongrowing branch, those exiting the growing branch and returning to the main axon, and those exiting the nongrowing branch to return to the main axon. The mean values shown indicate that mitochondria moving anterogradely and entering a branch point have more than twice the probability of sorting into the growing branch than the nongrowing branch, but mitochondria exit both branches at similar rates ( $n=14$ branched neurons).

an unstimulated branch, but those studies did not examine how the difference was generated (Goldberg and Schacher, 1987). To address this question, we selected hippocampal axons that had branched and used time-lapse microscopy to monitor the transport of mitochondria in the main axon and both branches. We first found that the growing branch does have a higher density of mitochondria than the nongrowing branch (Fig. 4). By tracking the movement of every mitochondrion that passed through the branch point during an interval of several hours, we found that anterogradely moving mitochondria from the main axon enter the growing branch twice as often as the nongrowing branch. At the same time, the frequency with which mitochondria exit from the growing versus nongrowing branch is not significantly different (Table 1). Thus, mitochondrial exit from nongrowing branches is not upregulated, but entry drops twofold. As a result, the constitutive rate of exit of mitochondria from both growing and nongrowing branches does slightly exceed the rate at which they enter nongrowing branches; thus, nongrowing branches show a net loss of mitochondria (Fig. 4). We saw no examples of the selective sorting of mitochondria into a nongrowing branch before its resumption of growth. Instead, the development of increased mitochondrial density in a growing branch followed its resumption of growth in all cases, sometimes by as much as 10 min. Thus, as for entry of mitochondria into nascent axons, their selective sorting into growing branches follows changes in axonal growth.
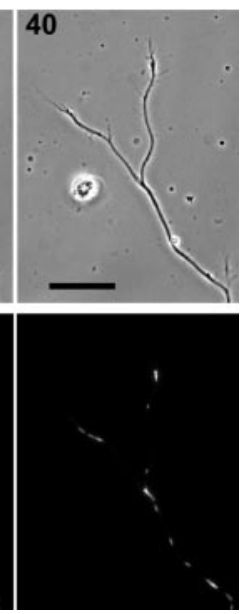

\section{Mitochondria are delivered into} growing processes by specific organelle motility rather than bulk axoplasmic flow

A simple explanation for the transport of mitochondria into a growing axon or axon branch would be that it follows a bulk flow of axoplasm into the growing region. Bulk flow has been suggested to underlie axon formation and the movement of organelles into the nascent axon (Bradke and Dotti, 1997). Although a coherent column of axoplasm probably does not flow into axons, it is clear that axonal elongation is supported by the abundant anterograde transport of organelles and proteins from the soma. However, in peripheral neurons, the delivery of mitochondria to the growing tip of an axon involves a complex interplay of anterograde and retrograde movements and does not suggest a nonspecific flow of organelles or axoplasm (Morris and Hollenbeck, 1993, 1995). To test the bulk axoplasmic flow hypothesis in hippocampal neurons, we produced rapid elongation of minor processes by applying mechanical tension to the tip with a glass needle (Lamoureux et al., 2002), a technique we will refer to as "towing." Elongation of a process by towing results in the construction of a normal axon but without the involvement of a growth cone (Bray, 1984). We visualized mitochondria throughout periods of axonal towing and asked whether they were recruited into these axons as they would be for naturally growing ones. Our results showed that in contrast to the response of mitochondrial traffic to normal axonal elongation, there was no rapid movement of mitochondria into any of the five processes that we towed. They did not develop the high mitochondrial density at the distal end that would be expected during growth cone-mediated elongation (Fig. 5) (Morris and Hollenbeck, 1993). After a delay of 10-30 min or longer, we sometimes observed substantial movement of mitochondria into a towed process (Fig. 5B, 124 min time point). This is probably attributable to spontaneous growth rather than towing because after a protracted period of towing, many processes developed a large and highly motile growth cone and reestablished contact with the substratum (Lamoureux et al., 2002). It was only after the reformation of a growth cone that mitochondrial movement into the towed axons was observed (Fig. 5B, $124 \mathrm{~min}$ ). In further contradiction of the bulk flow hypothesis, we occasionally observed retrograde movement of mitochondria within a process that was being towed (e.g., Fig. 5A), as is also seen during growth cone-mediated outgrowth of peripheral neurons (Morris and Hollenbeck, 1993).

Our data give some suggestion that the clustering of mitochondria at a growing tip and sorting at a branch point into the growing branch occur via different mechanisms (Morris and Hollenbeck, 1993) (Fig. 4, Table 1). Therefore, we used mechanical towing of an axonal branch to ask whether mitochondrial sorting at branch points of established axons could be driven by bulk axoplasmic flow or whether it too was dependent on specific mitochondrial transport. We found that towing one of two sister branches of a hippocampal axon was not accompanied by a streaming of mitochondria into the process (a representative example of the six branches towed is shown in Fig. 6). Thus, towed axon growth, which recruits the components of normal axoplasm 
into the new process (Bray, 1984), does not result in the rapid movement of mitochondria into the axon. We conclude that mitochondrial movement from the soma into a growing axon, and from an axon into a growing branch, results from specific, directed motility rather than bulk flow of axoplasm or organelles.

Growth cone activity is not necessary for mitochondrial sorting into growing branches

An active growth cone recruits mitochondria into the distal region of a growing axon and generates their characteristic distal accumulation in both peripheral (Morris and Hollenbeck, 1993) and central (Figs. 1, 2, 4, 5) neurons. However, it is possible that preferential sorting of mitochondria at branch points occurs by a different mechanism or responds to a different signal. Therefore, we tested whether sorting into the actively growing branch also requires an active growth cone at the tip of the growing branch, or whether elongation alone can drive it. When hippocampal neurons are grown in cytochalasin E, which disrupts F-actin and eliminates growth cones, branched axons nonetheless display alternating periods of growth (Ruthel and Hollenbeck, 2000). Using time-lapse microscopy, we tracked the movement of R123-labeled mitochondria through the branch points of five neurons growing in cytochalasin $\mathrm{E}$ and quantified movements into and out of growing and quiescent branches (Fig. 7). We found that a mean of 5.60 mitochondria/hr entered the growing branch, versus $0.68 / \mathrm{hr}$ for the nongrowing branch ( $p=0.025$, paired $t$ test); $3.29 / \mathrm{hr}$ exited the growing branch, whereas $2.02 / \mathrm{hr}$ exited the nongrowing branch ( $p=0.225$, paired $t$ test). These data indicate that an active growth cone is not necessary for mitochondria to sort very specifically at branch points into the elongating branch.

\section{Discussion}

The functions and products of mitochondria are required most acutely in different regions of the neuron at different times during development. Thus, neurons must control the traffic of mitochondria to reposition them according to their current needs. Although little is known about how mitochondrial traffic is organized during development, it is clear that neurons undergo not only persistent, long-term modifications involving altered patterns of gene expression but also more rapid and reversible modifications that involve changes in the cytoskeleton and organelle traffic (Gallo and Letourneau, 2000; Huang and Reichardt, 2001). Interrelated modifications of neuronal structure and function, such as axon outgrowth and the repositioning of mitochondria, may occur as responses to the same set of intracellular signals, or one event may actually give rise to another. In the first part of this study, we tested and rejected the hypothesis that the movement of mitochondria into specific regions of hippocampal neurons plays a causative role in axonal determination and elongation.

A

B
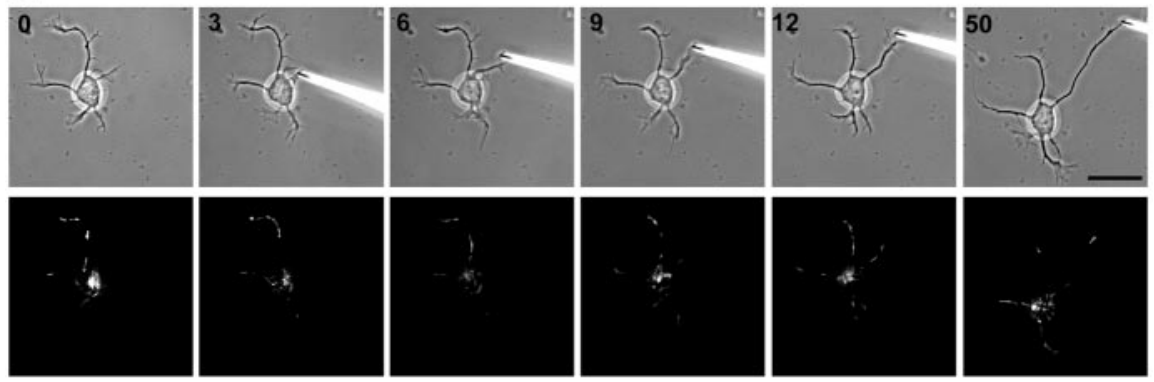

Figure 5. Towed growth of a minor process does not induce selective mitochondrial entry or clustering near the tip $(n=5)$. body where mitochondria were clustered. There was no immediate entry of mitochondria into the process as tension was applied

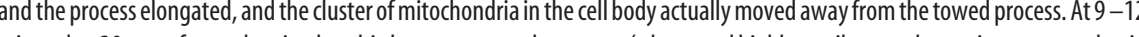
present has vacated the process during the application of tension. However, there are numerous mitochondria in the towed process by $124 \mathrm{~min}$, after a growth cone has been established. Scale bar, $20 \mu \mathrm{m}$.

\section{Changes in mitochondrial transport do not precede changes in axonal growth}

Our data make it clear that the orientation of somatic mitochondria toward the base of a minor process neither precedes nor is required for the development of that process into an axon. Nearly two-thirds of axons form without the somatic mitochondrial cluster being located near their base (Fig. 1), and mitochondria enter the axon regardless of their relative positions, just as efficiently as in any other minor process during a period of elongation (Fig. 2). The establishment of a higher density of mitochondria does not precede axonal determination and elongation because the mitochondrial density of the axon actually changes little and remains lower than that of the other minor processes until well into its outgrowth (Fig. 3). After axonal elongation has begun in earnest, a reduction in the mitochondrial density of the other processes occurs, and this may be an element of the suppression of the formation of supernumerary axons. If so, then mitochondrial traffic may provide a more fundamental indicator that axonal identity has been established than the relative length of the processes.

It was notable that the average mitochondrial density of a nascent axon was actually substantially lower than the average for the other minor processes in the initial measurement (when the future axon was still of a length comparable with that of the minor processes). This is probably because the processes grow in a cyclical manner, alternating between periods of growth and pauses or retractions (Ruthel and Banker, 1999). In our contin- 

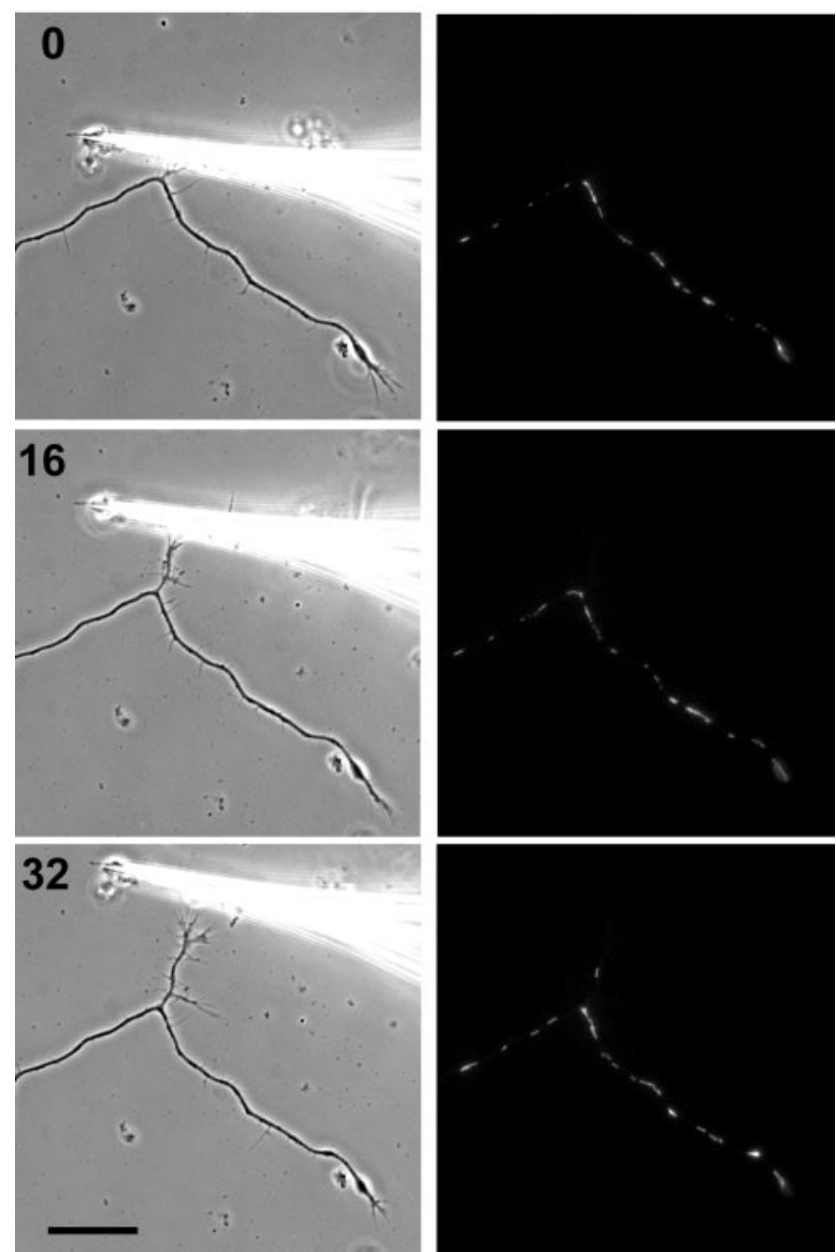

Figure 6. Mitochondrial transport into axonal branches does not respond to towed branch growth. A branched axon is shown in phase contrast and R123 fluorescence images at 16 min intervals. Tension was applied to the upper of the two branches using a glass needle. Despite the presence of numerous mitochondria in the parent axon around the branch point, there is no immediate rush of mitochondria into the towed branch. Even after $16 \mathrm{~min}$ of applied tension and $>10 \mu \mathrm{m}$ of growth, no mitochondria enter the branch. By $32 \mathrm{~min}$ and $20 \mu \mathrm{m}$ of growth, a single mitochondrion can be seen in the towed branch. Scale bar, $20 \mu \mathrm{m}$.

uous measurement protocol, this meant that when we quantified mitochondrial density in a process just before the sustained growth that occurs during axon formation, we were likely to have caught that process in a period of relative inactivity. The cycles of growth and retraction in minor processes are associated with net redistributions of mitochondria, such that mitochondria fill processes undergoing bursts of elongation and vacate those that are retracting or paused; this can be seen in the unmanipulated processes of the cell shown in Figure $5 A$.

During alternating growth of axonal branches, the changes in mitochondrial density that we observed also followed rather than preceded the resumption of axonal elongation (Fig. 4). Thus, as for axonal determination, it does not seem possible that a shift in the distribution of mitochondria could cause any of the subsequent events of branch elongation. These data are consistent with those from unbranched peripheral neurons (Morris and Hollenbeck, 1993). They are also consistent with the nature of mitochondrial transport in neurons: the saltatory, bidirectional nature of movement precludes very rapid changes in their overall distribution (Hollenbeck, 1996) and makes them more likely to respond to signals than to cause them. Axonal mitochondria undergo redistribution after, and probably in response to, changes in axonal growth.
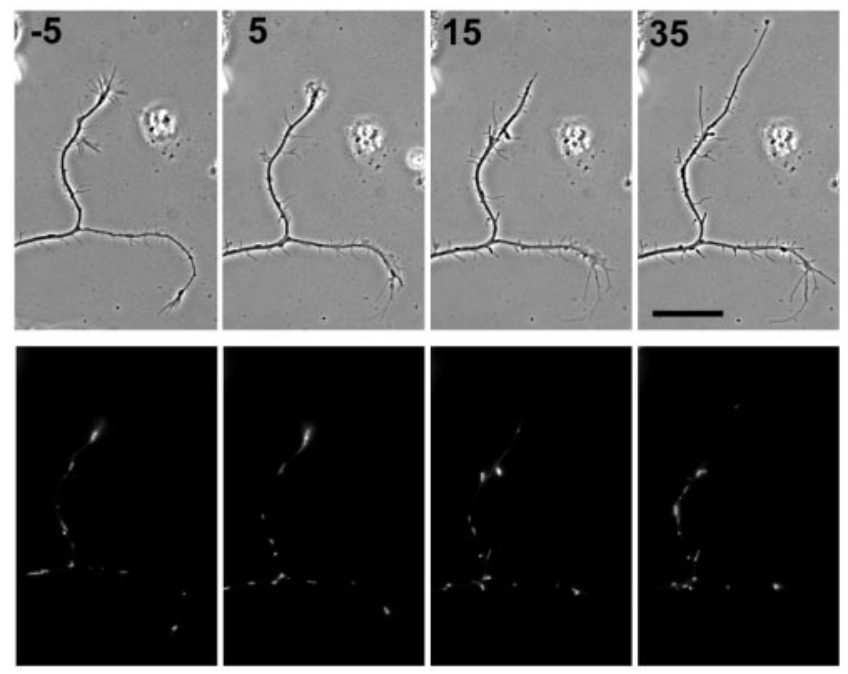

Figure 7. Mitochondrial sorting in axon branches after disruption of growth cones by cytochalasin E. Phase contrast and R123 fluorescence images are shown for a branched axon $5 \mathrm{~min}$ before $(-5)$ and 5, 15, and 35 min after application of cytochalasin E. By $15 \mathrm{~min}$, the branch oriented toward the top of the page but not the other branch has begun to elongate in the absence of growth cone motility. Mitochondria continue to sort selectively into the growing branch, but note that there is no longer an accumulation of mitochondria at the growing tip as there was before disruption of the growth cones. Scale bar, $20 \mu \mathrm{m}$.

\section{Mitochondrial transport into elongating axons: regulated} organelle transport or bulk flow of cytoplasmic components? To experimentally separate the elongation of the axon from the activity of the growth cone, we towed hippocampal axons by applying mechanical tension to the tip. Axons generated by towing are supplied with a normal complement of cytoskeletal proteins and organelles (Bray, 1984). If the delivery of mitochondria to an elongating axon occurred through a general anterograde flow of axoplasm (Bradke and Dotti, 1997), then they should enter and accumulate in a towed axon to the same extent as one advancing by growth cone activity. In fact, we found that towed axons not only fail to attain the same overall density of mitochondria as normally growing axons but also do not develop the typical high concentration of mitochondria near the tip. This indicates that the constitutive anterograde flow of organelles and proteins is not responsible for the selective delivery of mitochondria into growing axons. Even if a generalized pattern of cytoplasmic flow did occur in axons, the bidirectional nature of mitochondrial movement would likely preclude them from participating. The same is likely to be the case for other bidirectionally transported classes of axonal organelles such as endosomes and autophagic vacuoles (Hollenbeck, 1993; Overly et al., 1996).

It is possible that an axon with a reduced or absent growth cone has lower energy requirements and therefore does not require a high density of mitochondria. However, such an axon could also initially fail to generate the intracellular signal(s) that normally stimulates mitochondrial entry and accumulation in the distal region. Indeed, we only observed substantial recruitment of mitochondria in towed axons after instances of spontaneous elaboration of the growth cone and reattachment to the substratum.

\section{What fundamental mechanisms control mitochondrial redistribution in axons?}

Mitochondrial transport in axons is coordinated with axonal outgrowth in two different ways. First, in the presence, and only in the presence, of an active growth cone, mitochondria enter the axon in large numbers and are recruited to the distal region. 
When axons elongate in the absence of growth cones, under cytochalasin treatment or by towing, they do not recruit mitochondria to the distal region. Second, even in the absence of active growth cones, mitochondria moving anterogradely along an axon and, reaching a branch point, sort preferentially into the growing branch. This occurs even when the branch point is located at a considerable distance from the tips of the branches. However, it does not occur when an axon branch elongates by towing. These data suggest that the intracellular signals for the two regulatory events, distal accumulation and sorting at branch points, are distinct or arise in different ways.

We propose that the recruitment of mitochondria into a newly formed axon and their concentration in the distal region as the axon grows are stimulated by signaling that arises in the growth cone region or other distal regions in vivo and affects the motility behavior of mitochondria. When mitochondria respond to the activity of the growth cone, their anterograde motor activity and overall motility duty ratio are specifically altered with respect to their distance from the growth cone (Morris and Hollenbeck, 1993). Because the distal axon is a focus of signaling activity, the local stimulation of specific kinase pathways could play a role in modulating mitochondrial behavior there (S. R. Chada and P. J. Hollenbeck, unpublished observations). In the absence of an active growth cone or synapse, the magnitude of signaling activity in the distal axon would be diminished, and mitochondria would not be recruited into the region. The recruitment of mitochondria into the region of an active growth cone is clearly important for sustained outgrowth because actin polymerization and depolymerization, which are concentrated there, consume an astonishing fraction the total ATP of the neuron (Bernstein and Bamburg, 2003).

In the case of branch points, we suggest that sorting of mitochondria arises through reduction in the microtubule density of the nongrowing branch. Fewer microtubules per unit of crosssectional area would mean fewer available tracks leading from the main axon through the branch point and into the nongrowing branch. This would produce the result that we see: fewer mitochondria that reach the branch point enter the nongrowing branch than the growing branch. However, because mitochondria moving within the nongrowing axon are already engaged with a microtubule track, their exit from the branch would be neither impeded nor stimulated, and this is the result that we obtained. During alternating branch growth, the nongrowing branch is uniformly observed to be significantly thinner than the growing branch (Ruthel and Banker, 1999; Ruthel and Hollenbeck, 2000), supporting the idea that it may contain a more sparse cytoskeleton and fewer tracks for organelle movement.

\section{References}

Ankarkrona M, Dypbukt JM, Bonfococ E, Zhivotovsky B, Orrenius S, Lipton SA, Nicotera P (1995) Glutamate-induced neuronal death: a succession of necrosis or apoptosis depending on mitochondrial function. Neuron 15:961-973.

Bernstein BW, Bamburg JR (2003) Actin-ATP hydrolysis is a major energy drain for neurons. J Neurosci 23:1-6.

Berthold CH, Fabricius C, Rydmark M (1993) Axoplasmic organelles at the nodes of Ranvier, I: occurrence and distribution in large myelinated spinal roots of the adult cat. J Neurocytol 22:925-940.

Bindokas VP, Lee CC, Colmers WF, Miller RJ (1998) Changes in mitochondrial function resulting from synaptic activity in the rat hippocampal slice. J Neurosci 18:4570-4587.

Bottenstein JE, Sato GH (1979) Growth rate of a neuroblastoma cell line in a serum-free supplemented medium. Proc Natl Acad Sci USA 96:514-519.
Bradke F, Dotti CG (1997) Neuronal polarity: vectorial cytoplasmic flow precedes axon formation. Neuron 19:1175-1186.

Bray D (1984) Axonal growth in response to experimentally applied mechanical tension. Dev Biol 102:379-389.

Bristow EA, Griffiths PG, Andrews RM, Johnson MA, Turnbull DM (2002) The distribution of mitochondrial activity in relation to optic nerve structure. Arch Ophthamol 120:791-796.

Dotti CG, Banker GA (1991) Intracellular organization of hippocampal neurons during the development of neuronal polarity. J Cell Sci [Suppl] 15:75-84.

Dotti CG, Sullivan CA, Banker GA (1988) The establishment of polarity by hippocampal neurons in culture. J Neurosci 8:1454-1468.

Gallo G, Letourneau PC (2000) Neurotrophins and the dynamic regulation of the neuronal cytoskeleton. J Neurobiol 44:159-173.

Goldberg DJ, Schacher S (1987) Differential growth of branches of a regenerating bifurcate axon is associated with differential axonal transport of organelles. Dev Biol 124:35-40.

Goslin K, Banker G (1991) Rat hippocampal neurons in low density culture. In: Culturing nerve cells (Banker G, Goslin K, eds), pp 252-281. Cambridge, MA: MIT.

Hollenbeck PJ (1993) Products of endocytosis and autophagy are retrieved from axons by regulated retrograde organelle transport. J Cell Biol 121:305-315.

Hollenbeck PJ (1996) The pattern and mechanism of mitochondrial transport in axons. Front Biosci 1:d91-d102.

Huang EJ, Reichardt LF (2001) Neurotrophins: roles in neuronal development and function. Annu Rev Neurosci 24:677-736.

Kageyama GH, Wong-Riley M (1982) Histochemical localization of cytochrome oxidase in the hippocampus: correlation with specific neuronal types and afferent pathways. Neuroscience 7:2337-2361.

Kosel S, Hofhaus G, Maassen A, Vieregge P, Graeber MB (1999) Role of mitochondria in Parkinson disease. Biol Chem 380:865-870.

Lamoureux P, Buxbaum RE, Heidemann SR (1998) Axonal outgrowth of cultured neurons is not limited by growth cone competition. J Cell Sci 111:3245-3252.

Lamoureux P, Ruthel G, Buxbaum RE, Heidemann SR (2002) Mechanical tension can specify axons in hippocampal neurons. J Cell Biol 159:499-508.

Mattson MP, Partin J (1999) Evidence for mitochondrial control of neuronal polarity. J Neurosci Res 56:8-20.

Morris RL, Hollenbeck PJ (1993) The regulation of bidirectional mitochondrial transport is coordinated with axonal outgrowth. J Cell Sci 104:917-927.

Morris RL, Hollenbeck PJ (1995) Axonal transport of mitochondria along microtubules and F-actin in living vertebrate neurons. J Cell Biol 131:1315-1326.

Nicholls DG, Budd SL (2000) Mitochondria and neuronal survival. Physiol Rev 80:315-360.

Overly CC, Rieff HI, Hollenbeck PJ (1996) Organelle motility and metabolism in axons vs dendrites of cultured hippocampal axons. J Cell Sci 109:971-980.

Raha S, Robinson BH (2000) Mitochondria, oxygen free radicals, disease and ageing. Trends Biochem Sci 25:502-508.

Reynolds IJ (1999) Mitochondrial membrane potential and the permeability transition in excitotoxicity. Ann NY Acad Sci 893:33-41.

Ruthel G, Banker G (1999) Role of moving growth cone-like "wave" structures in the outgrowth of cultured hippocampal axons and dendrites. J Neurobiol 39:97-106.

Ruthel G, Hollenbeck PJ (2000) Growth cones are not required for initial establishment of polarity or differential axon branch growth in cultured hippocampal neurons. J Neurosci 20:2266-2274.

Sawa A (2001) Mechanisms for neuronal cell death and dysfunction in Huntington's disease: pathological cross-talk between the nucleus and the mitochondria? J Mol Med 79:375-381.

Swerdlow RH, Kish SJ (2002) Mitochondria in Alzheimer's disease. Int Rev Neurobiol 53:341-385.

Waxman SG, Ritchie JM (1993) Molecular dissection of the myelinated axon. Ann Neurol 33:121-136.

Wong-Riley M, Welt C (1980) Histochemical changes in cytochrome oxidase of cortical barrels after vibrissal removal in neonatal and adult mice. Proc Natl Acad Sci USA 77:2333-2337. 